\title{
ANÁLISE DOS CASOS NOTIFICADOS DE TUBERCULOSE NA REGIÃO CENTRO-OESTE
}

\section{ANALYSIS OF REPORTED CASES OF TUBERCULOSIS IN THE MIDWEST REGION}

\section{ANÁLISIS DE LOS CASOS NOTIFICADOS DE TUBERCULOSIS EN LA REGIÓN DEL MEDIO OESTE}

\author{
Yasmin Souza Silva ${ }^{1}$ \\ Paulienne Ramos da Silva Matias ${ }^{2}$ \\ Lucíola Silva Sandim ${ }^{3}$ \\ Maria Goretti Queiroz ${ }^{4}$ \\ Jeovana Romero de Serqueira ${ }^{5}$
}

Como citar este artigo: Silva YS, Matias PRS, Sandim LS, Queiroz MG, Serqueira JR. Análise dos casos notificados de tuberculose na região Centro-Oeste. Rev baiana enferm. 2022;36:e43082.

\begin{abstract}
Objetivo: analisar a caracterização dos casos de tuberculose notificados na região Centro-Oeste, no período de 2010 a 2019. Método: estudo ecológico, longitudinal, que utilizou, para análise estatística, o programa Microsoft Excel 2010 (cálculo das frequências) e o teste Chi quadrado (X2) (verificar se as frequências diferiam). Resultados: observou-se maior prevalência da tuberculose em pacientes do sexo masculino $(70,8 \%)$, pertencentes à faixa etária entre 25-34 anos (23,6\%), da raça/cor parda (53\%), moradores da zona urbana (83\%) e que não tiveram sua escolaridade declarada (28,6\%). Ao considerar aspectos epidemiológicos, destacaram-se casos novos (82,6\%), na forma pulmonar $(85,6 \%)$, com soropositividade negativa ao HIV $(60,4 \%)$ e que não tiveram declarado o hábito de tabagismo $(51,1 \%)$. Conclusão: os casos de tuberculose notificados na região Centro-Oeste assemelham-se aos encontrados na literatura, reforçando que a tuberculose é uma doença infecciosa atual e sua erradicação ainda exige muito trabalho e políticas públicas efetivas.
\end{abstract}

Descritores: Tuberculose. Epidemiologia. Incidência. Perfil de Saúde. Sistemas de Informação.

Objective: to analyze the characterization of tuberculosis cases reported in the Midwest region, in the period from 2010 to 2019. Method: ecological, longitudinal study, which used, for statistical analysis, the Microsoft Excel 2010 program (calculation of frequencies) and the Chi square test $\left(X^{2}\right)$ (check if the frequencies differed). Results: a higher prevalence of tuberculosis was observed in male patients (70.8\%), aged between 25-34 years (23.6\%), mixed race/color (53\%), urban residents (83\%) and who did not have their schooling declared (28.6\%). When considering epidemiological aspects, new cases (82.6\%), in the pulmonary form (85.6\%), with HIV-negative seropositivity (60.4\%) and who had not declared smoking (51.1\%) were highlighted. Conclusion: the cases of tuberculosis reported in the Midwest region are similar to those found in the literature, reinforcing that tuberculosis is a current infectious disease and its eradication still requires a lot of work and effective public policies.

Descriptors: Tuberculosis. Epidemiology. Incidence. Health Profile. Information Systems.

\footnotetext{
Estudante de Enfermagem. Centro Universitário de Goiatuba (UniCerrado). Goiatuba, Goiás, Brasil. https://orcid.org/0000-000 I-8250-I 287.

Enfermeira. Docente do Centro Universitário de Goiatuba (UniCerrado). Goiatuba, Goiás, Brasil. paulienneramos@yahoo.com.br. https://orcid.org/0000-00031980-6154

Enfermeira. Mestre em Enfermagem. Docente do Centro Universitário de Goiatuba (UniCerrado). Goiatuba, Goiás, Brasil. https://orcid.org/0000-0002-654 I-00 I 4.

Odontóloga. Doutora em Educação. Docente da Universidade Federal de Goiás. Goiânia, Goiás, Brasil. https://orcid.org/0000-0002-7363-4835.

Enfermeira. Docente do Centro Universitário de Mineiros. Mineiros, Goiás, Brasil. https://orcid.org/0000-000 I-6721-5151.
} 
Objetivo: analizar la caracterización de los casos de tuberculosis reportados en la región Medio Oeste, en el período de 2010 a 2019. Método: estudio ecológico, longitudinal, que utilizó, para el análisis estadístico, el programa Microsoft Excel 2010 (cálculo de frecuencias) y la prueba de Chi cuadrado ( $X^{2}$ ) (comprobar si las frecuencias diferían). Resultados: se observó una mayor prevalencia de tuberculosis en pacientes varones (70,8\%), con edades comprendidas entre 25-34 años (23,6\%), mestizos/color (53\%), residentes urbanos (83\%) y a quienes no se les declaró la escolaridad (28,6\%). Al considerar los aspectos epidemiológicos, se destacaron los nuevos casos (82,6\%), en la forma pulmonar (85,6\%), con seropositividad VIH negativa (60,4\%) y que no habian declarado fumar (51, 1\%). Conclusión: los casos de tuberculosis reportados en la región del Medio Oeste son similares a los encontrados en la literatura, reforzando que la tuberculosis es una enfermedad infecciosa actual y su erradicación aún requiere mucho trabajo y políticas públicas efectivas.

Descriptores: Tuberculosis. Epidemiología. Incidencia. Perfil de Salud. Sistemas de Información.

\section{Introdução}

A tuberculose (TB) é uma doença infectocontagiosa, que ainda se configura como um problema de saúde pública no Brasil e no mundo. A taxa de incidência global da doença vem diminuindo $1,4 \%$ ao ano, porém, ao contrário do que se pensa, ainda é insuficiente para o alcance da meta da Estratégia da Organização Mundial da Saúde (OMS) pelo fim da TB. De acordo com essa instituição, é necessária uma taxa de redução global de 4 a 5\% ao ano para atingir menos de 10 casos por 100 mil habitantes até o ano de $2035^{(1-3)}$.

Nos últimos anos, muito foi feito, no Brasil, para combater a TB, porém ainda existem desafios a serem superados, para que se alcance o objetivo de tirar a doença dessa lista de problemas de saúde pública. Como exemplos desses desafios, tem-se, entre outros, a manutenção do enfrentamento da TB na agenda política, o fortalecimento das ações de controle da TB nas populações mais vulneráveis, a abordagem da doença na perspectiva dos determinantes sociais e a melhoria dos indicadores de desfecho do tratamento ${ }^{(4)}$.

De acordo com o Ministério da Saúde (MS), ocorrem anualmente no mundo em torno de 8 milhões de casos novos da doença e quase 3 milhões de pessoas morrem vitimadas por ela. Estatísticas mostram que, somente no ano de 2016, foram notificados 69.509 casos novos da doença. Segundo a OMS, o Brasil é considerado prioritário para o controle da doença no mundo, pois continua entre os 30 países de alta carga para TB e para coinfecção TB-HIV ${ }^{(1,5)}$.

É interessante ressaltar que, no Brasil, a TB teve um total de 877.509 casos confirmados no período de 2010 a 2019, permanecendo insatisfatórios os indicadores epidemiológicos, pois, em muitas regiões, houve aumento dos casos. Isso pode ser justificado pela piora no quadro sanitário nos últimos anos ou mesmo por uma melhora na capacidade de diagnóstico. Exemplo disso é a região Norte que, em 2010, apresentou um registro de 8.376 casos novos confirmados, enquanto que, em 2019, esse número subiu para 11.438. A região Sudeste apresentou aumento semelhante, tendo registrado, no ano de 2010, 38.375 novos casos e, em 2019, $40.355^{(6)}$.

Segundo as orientações da OMS, a TB ainda está longe de ser erradicada. Em decorrência do contínuo aumento no número de casos nos últimos anos, foram implementados planos para o controle da doença em todos os países, abordando a vacinação, a detecção precoce dos casos, a adesão ao tratamento, o controle de contatos, além da implantação de medidas de controle de infecção nas unidades de saúde e ações estratégicas para situações e populações especiais ${ }^{(7)}$.

A literatura consultada possibilitou observar-se que, para alcançar as metas de controle da TB, é imprescindível que se tenha a compreensão da epidemiologia da região, o que possibilitará identificar os grupos de populações mais vulneráveis e, dessa forma, facilitar a 
detecção precoce de novos casos. Este fato, inclusive, justifica a pesquisa desenvolvida.

Diante do exposto, este estudo objetivou analisar a caracterização dos casos de TB notificados na região Centro-Oeste, no período de 2010 a 2019.

\section{Método}

Trata-se de um estudo ecológico, longitudinal, de caráter quantitativo, que teve como área de pesquisa a região Centro-Oeste. A busca de dados foi realizada em 2020.

O período escolhido (2010-2019) justifica-se pela atuação da Atenção Básica em Saúde (ABS) nos últimos anos, que tem sido insatisfatória em relação ao acesso de primeiro contato, ao enfoque familiar e à orientação comunitária ${ }^{(8)}$. Essas ocorrências podem interferir diretamente nas ações de atenção à saúde, podendo justificar o aumento dos casos de TB nos últimos 10 anos.

As unidades de análise do estudo foram os estados de Goiás, Mato Grosso, Mato Grosso do Sul e Distrito Federal. A área de estudo ocupa 1.606.316,665 km² e possui uma população estimada em 16.504.303 milhões de habitantes para 2020, o que representa aproximadamente $7,8 \%$ da população do Brasil ${ }^{(9)}$.

Todos os dados foram extraídos do TabNet, plataforma Web do Departamento de Informática do Sistema Único de Saúde (DATASUS), tendo sido incluídos os casos de TB notificados na região Centro-Oeste, nos anos de 2010 a 2019. Os dados populacionais, para cálculo da taxa de incidência, foram obtidos com base nos dados disponibilizados pelas estimativas do Instituto Brasileiro de Geografia e Estatística (IBGE).

Para análise estatística, foi criado um banco de dados no programa Microsoft Excel 2010. Foram analisados os casos notificados por ano na Região e nos três estados, além do Distrito Federal, e calculados os valores percentuais das frequências de ocorrência dos casos de TB, para descrever as diferentes categorias investigadas: Análise Sociodemográfica (sexo, faixa etária, raça, escolaridade e região domiciliar) e Análise Epidemiológica (tabagismo, forma clínica, soropositividade ao HIV e tipo de entrada no Sistema de Saúde).

Além disso, o teste Chi quadrado $\left(\mathrm{X}^{2}\right)$ foi utilizado para verificar, nas categorias mencionadas, se as frequências de ocorrência diferiam entre as classes de grupamento dos dados. Nessas análises, não foi considerada a classe "não declarado". No tocante à soropositividade para o HIV, não foram também consideradas as classes "em andamento" e "não realizada". Todas as análises foram feitas utilizando-se o programa estatístico SYSTAT.

Por se tratar de análise de dados de domínio público, não houve necessidade de autorização de Comitê de Ética em Pesquisa para a realização da pesquisa.

\section{Resultados}

No período de 2010 a 2019, foram notificados 42.118 casos de TB na região Centro-Oeste, resultando em uma média de 4.212 casos por ano (Tabela 1).

Tabela 1 - Casos de tuberculose na região Centro-Oeste, por estados. Goiás, Mato Grosso, Mato Grosso do Sul e Distrito Federal, Brasil - 2010-2019. (N=42.118)

\begin{tabular}{l|c|c|c|c|c}
\hline Ano de Notificação & $\begin{array}{c}\text { Região Centro- } \\
\text {-Oeste }\end{array}$ & $\begin{array}{c}\text { Mato Grosso } \\
\text { do Sul }\end{array}$ & Mato Grosso & Goiás & $\begin{array}{c}\text { Distrito } \\
\text { Federal }\end{array}$ \\
\hline 2010 & 3.757 & 943 & 1.368 & 1.016 & 430 \\
2011 & 3.896 & 1.045 & 1.371 & 1.053 & 427 \\
2012 & 4.308 & 1.091 & 1.605 & 1.058 & 554 \\
2013 & 4.529 & 1.098 & 1.886 & 1.072 & 473 \\
2014 & 4.295 & 998 & 1.794 & 991 & 512 \\
2015 & 4.018 & 1.003 & 1.442 & 1.095 & 478 \\
2016 & 4.055 & 1.124 & 1.448 & 1.022 & 461 \\
2017 & 4.160 & 1.145 & 1.430 & 1.167 & 418 \\
2018 & 4.563 & 1.458 & 1.415 & 1.214 & 476 \\
2019 & 4.537 & 1.395 & 1.511 & 1.156 & 475 \\
\hline
\end{tabular}

Fonte: Elaboração própria. 
Analisando-se e comparando-se os anos de 2010 e 2019, encontrou-se um aumento na taxa de incidência de TB, tanto na região Centro-Oeste como no Brasil (Gráfico 1), não sendo esse aumento significativo. Entretanto, esse número ainda é preocupante, pois, mesmo com a implantação de estratégias de controle pela OMS e pelo MS brasileiro, os casos continuam aumentando na região Centro-Oeste e em todo o país.

Gráfico 1 - Taxa de incidência de tuberculose (por 1.000 habitantes). Brasil e região Centro-Oeste $-2010 / 2019$

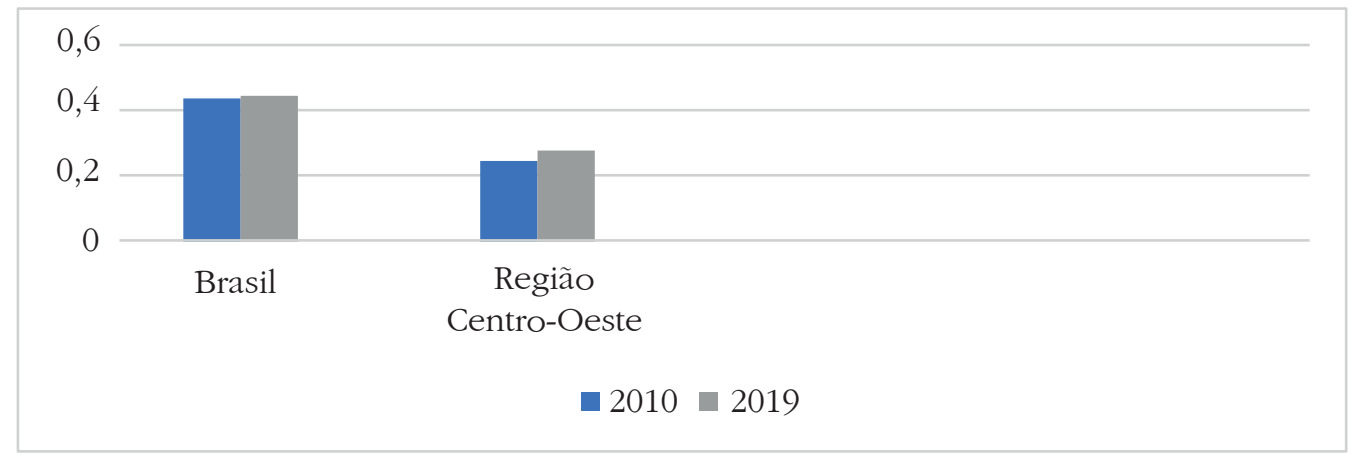

Fonte: Elaboração própria.

A incidência de TB na região Centro-Oeste foi significativamente maior entre homens do que entre mulheres, de acordo com o teste Chi-quadrado $\left(X^{2}=1.520 ; \mathrm{p}<0,001 ; \mathrm{gl}=1\right)$ e esta tendência ocorreu em todos os estados e no Distrito Federal, variando de 66,8\% no Distrito Federal a 75,1\% no Mato Grosso do Sul. Apenas o sexo de cinco pessoas não foi identificado na amostra de 42.118 casos reportados (Tabela 2).

As frequências da incidência da TB diferiram significativamente nas diferentes faixas etárias $\left(X^{2}=7.793 ; \mathrm{p}<0,001 ; \mathrm{gl}=6\right)$. As que apresentaram os maiores números de casos de TB foram aquelas de 25 a 34 anos e de 35 a 44 anos. A faixa etária com a menor frequência de ocorrência foi a de 0 a 14 anos. O padrão descrito para as maiores e menores frequências de casos de TB nas diferentes faixas etárias foi observado em todos os estados da região Centro-Oeste e no Distrito Federal (Tabela 2).

Foram encontradas diferenças estatísticas significativas no número de casos de tuberculose entre as distintas raças $\left(X^{2}=39.597 ; \mathrm{p}<0,001\right.$; $\mathrm{gl}=4)$. Os pardos apresentaram as maiores frequências, representando mais da metade do total de casos, seguidos pelos brancos. Os amarelos foram os menos frequentes. A frequência de casos de TB entre as distintas raças seguiu um padrão homogêneo em todos os estados da região Centro-Oeste e no Distrito Federal. É importante ressaltar que, em 2.199 casos, não foi identificada ou declarada a raça da pessoa com TB nas notificações (Tabela 2).

A escolaridade predominante foi de pessoas que não completaram o ensino fundamental $\left(\mathrm{X}^{2}=9.843 ; \mathrm{p}<0,001 ; \mathrm{gl}=8\right)$, especialmente aqueles que cursaram da $5^{\underline{a}}$ à $7^{\text {a }}$ séries ou da $1^{\underline{a}}$ à $3^{\underline{a}}$ séries. As menores frequências foram encontradas entre aquelas com curso superior incompleto. Novamente, foi observada homogeneidade entre os estados e o Distrito Federal quanto ao padrão para as maiores e menores frequências de ocorrência de TB por nível de escolaridade. Entretanto, em um número expressivo de casos, não foi feita essa identificação ou foi declarado "não se aplica" (Tabela 2). 
Tabela 2 - Perfil sociodemográfico de pacientes com tuberculose por estados. Região Centro-Oeste e Distrito Federal, Brasil - 2010-2019. $(\mathrm{N}=42.118)$

\begin{tabular}{|c|c|c|c|c|c|c|}
\hline Variáveis do Perfil(1) & $\begin{array}{c}\text { Mato Grosso } \\
\text { do Sul }\end{array}$ & $\begin{array}{c}\text { Mato } \\
\text { Grosso }\end{array}$ & Goiás & $\begin{array}{l}\text { Distrito } \\
\text { Federal }\end{array}$ & Total & $\%$ \\
\hline \multicolumn{7}{|l|}{ Sexo } \\
\hline Masculino & 8.469 & 10.397 & 7.808 & 3.180 & 29.854 & 70,8 \\
\hline Feminino & 2.797 & 4.863 & 3.021 & 1.578 & 12.259 & 29,1 \\
\hline Não Declarado & 2 & 2 & 1 & - & 5 & $<0,1$ \\
\hline Probabilidade & $\mathrm{p}<0,001$ & & & & & \\
\hline \multicolumn{7}{|l|}{ Faixa Etária } \\
\hline $0-14$ & 366 & 767 & 179 & 148 & 1.460 & 3,5 \\
\hline $15-24$ & 1.672 & 2.203 & 1.365 & 525 & 5.765 & 13,7 \\
\hline $25-34$ & 2.939 & 3.359 & 2.511 & 1.141 & 9.950 & 23,6 \\
\hline $35-44$ & 2.275 & 2.832 & 2.384 & 1.009 & 8.500 & 20,2 \\
\hline $45-54$ & 1.802 & 2.456 & 1.912 & 848 & 7.018 & 16,7 \\
\hline $55-64$ & 1.190 & 1.853 & 1.255 & 531 & 4.829 & 11,5 \\
\hline $65 \mathrm{ou}+$ & 1.024 & 1.792 & 1.224 & 556 & 4.596 & 10,8 \\
\hline Probabilidade & $\mathrm{p}<0,001$ & & & & & \\
\hline \multicolumn{7}{|l|}{ Raça } \\
\hline Branca & 3.260 & 3.173 & 2.482 & 1.184 & 10.099 & 24,0 \\
\hline Preta & 855 & 1.896 & 1.094 & 534 & 4.379 & 10,4 \\
\hline Amarela & 156 & 119 & 133 & 38 & 446 & 1,1 \\
\hline Parda & 4.796 & 8.495 & 6.500 & 2.544 & 22.335 & 53,0 \\
\hline Indígena & 1.366 & 1.233 & 41 & 20 & 2.660 & 6,3 \\
\hline Não declarada & 835 & 346 & 580 & 438 & 2.199 & 5,2 \\
\hline Probabilidade & $\mathrm{p}<0,001$ & & & & & \\
\hline \multicolumn{7}{|l|}{ Escolaridade } \\
\hline Analfabeto & 499 & 981 & 505 & 162 & 2.147 & 5,1 \\
\hline $1^{\underline{a}}$ à $3^{\underline{a}}$ & 1.534 & 2.329 & 1.548 & 488 & 5.899 & 14,0 \\
\hline $4^{\underline{a}}$ & 717 & 1.033 & 772 & 249 & 2.771 & 6,6 \\
\hline $5^{\underline{a}}$ à $7^{\underline{a}}$ & 1.892 & 2.645 & 1.818 & 635 & 6.990 & 16,6 \\
\hline $\begin{array}{l}\text { Fundamental } \\
\text { Completo }\end{array}$ & 724 & 1.177 & 722 & 272 & 2.895 & 6,9 \\
\hline Médio Incompleto & 778 & 1.274 & 725 & 322 & 3.099 & 7,3 \\
\hline Médio Completo & 757 & 1.773 & 1.081 & 497 & 4.108 & 9,7 \\
\hline Superior Incompleto & 136 & 305 & 172 & 125 & 738 & 1,8 \\
\hline Superior Completo & 216 & 591 & 276 & 344 & 1.427 & 3,4 \\
\hline Não Declarado & 4.015 & 3.154 & 3.211 & 1.664 & 12.044 & 28,6 \\
\hline Probabilidade & $\mathrm{p}<0,001$ & & & & & \\
\hline \multicolumn{7}{|l|}{ Região Domiciliar } \\
\hline Urbana & 8.780 & 12.668 & 9.597 & 3.887 & 34.932 & 83,0 \\
\hline Rural & 2.124 & 2.230 & 802 & 195 & 5.351 & 12,7 \\
\hline Periurbana & 79 & 76 & 66 & 82 & 303 & 0,7 \\
\hline Não Declarado & 285 & 288 & 365 & 594 & 1.532 & 3,6 \\
\hline Probabilidade & $\mathrm{p}<0,001$ & & & & & \\
\hline
\end{tabular}

Fonte: Elaboração própria.

Nota: Sinal convencional utilizado:

- Dado numérico igual a zero não resultante de arredondamento.

(1) Os valores de probabilidade referem-se às comparações feitas com os valores totais da região.

A TB acometeu, em significativo maior número, pessoas residentes na área urbana $\left(X^{2}=51.734\right.$; $\mathrm{p}<0,001 ; \mathrm{gl}=2$ ). Nas áreas rurais, a frequência de casos registrados foi bem menor. Esta tendência de distribuição do número de casos nas três áreas urbanas foi similar nos três estados investigados e no Distrito Federal (Tabela 2). 
Sob a ótica da análise clínico epidemiológica, foi possível observar que, em pouco mais da metade dos casos (51,1\%), a informação sobre o tabagismo não foi disponibilizada. Dentre os demais, a maioria declarou-se não fumante $(36,2 \%)$ e este padrão prevaleceu para todos os estados e no Distrito Federal. A frequência de fumantes foi significativamente maior do que a de não fumantes $\left(X^{2}=4739 ; \mathrm{p}<0,001 ; \mathrm{gl}=1\right)$.

A forma clínica predominante na área do estudo foi a pulmonar, $85,6 \%$ dos casos (36.073), com frequência significativamente maior do que as formas extrapulmonares $\left(X^{2}=52.659\right.$; $\mathrm{p}<0,001 ; \mathrm{gl}=2$ ). Esta prevalência ocorreu em todos os estados e no Distrito Federal. Apenas $2,8 \%$ dos casos (1.173) apresentaram ambas as formas de acometimento (pulmonar e extrapulmonar). Em cerca de 0,3\% não foi declarada a forma clínica da TB nos casos considerados neste estudo (Gráfico 2).

Gráfico 2 - Número de casos de tuberculose por forma clínica de acometimento e por estados. Região Centro-Oeste e Distrito Federal, Brasil - 2010-2019

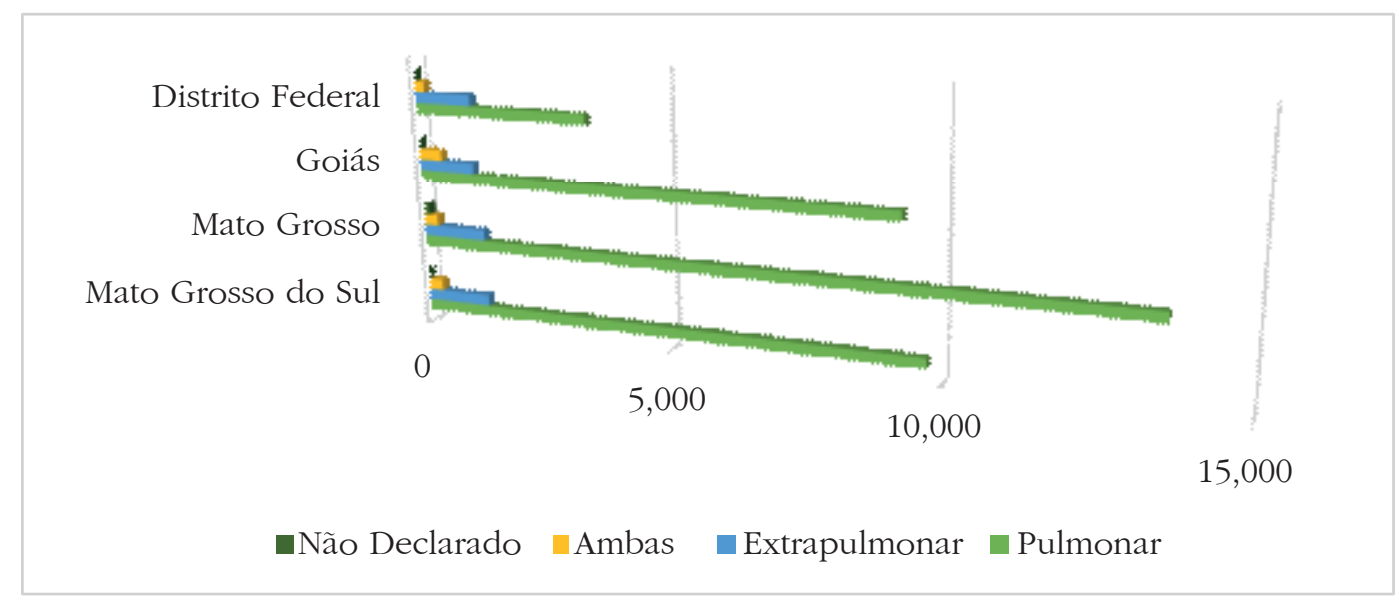

Fonte: Elaboração própria.

Com diferença estatisticamente significativa $\left(X^{2}=15.303 ; \mathrm{p}<0,001 ; \mathrm{gl}=1\right)$, a frequência da TB em pessoas com sorologia positiva para o HIV foi menor $(9,9 \%)$ do que a de casos testados negativos para o HIV (25,4\%). Cerca de 26,7\% (11.254 pessoas) não haviam realizado o teste de HIV e outros 0,3\% (115 pessoas) nada declararam sobre esta questão. Entretanto, 1.122 pessoas $(2,7 \%)$ aguardavam os resultados do teste de soropositividade para o HIV. A prevalência de casos negativos sobre os positivos para o HIV foi observada em todos os estados e no Distrito Federal.

Foram encontradas diferenças estatísticas significativas nas frequências dos diferentes tipos de entrada dos casos de TB no sistema de saúde da região Centro-Oeste $\left(X^{2}=77.759 ; \quad p<0,001\right.$; $\mathrm{gl}=4$ ). Os novos casos de TB foram os mais frequentes $(82,6 \%)$, constituindo maioria em todos os estados da região Centro-Oeste e no Distrito
Federal. Apenas o Distrito Federal apresentou número de casos transferidos ligeiramente maior (199) do que de recidiva (180). Nos demais estados da região, os casos de recidiva superaram os de transferência. A entrada pós-óbito foi a que apresentou menor frequência $(0,3 \%)$.

\section{Discussão}

Entre os anos de 2010 e 2019, foi notificado um total de 42.118 casos de TB na região Centro-Oeste, incluindo as formas pulmonares e extrapulmonares. Na Tabela 1, pode-se notar o crescente aumento do número de casos ao longo dos anos, o que pode levar ao entendimento de que ocorreram falhas no cuidado do paciente com TB e uma busca ativa deficiente dos sintomáticos. 
O ano de 2015, comparado aos anos anteriores, registrou pequena diminuição de casos de TB notificados na região Centro-Oeste. Pode ser levantada a hipótese de uma busca ativa mais intensa, quando equiparado aos demais anos. Entretanto, mesmo com essa pequena redução, os casos de TB tiveram um aumento significativo nos anos seguintes, configurando-se um grave problema de saúde pública.

Ao identificar as características sociodemográficas da região Centro-Oeste mostradas na Tabela 2, observou-se maior perfil do sexo masculino, com 70\%, dos casos pertencentes à faixa etária entre 25 e 34 anos, com 23,6\%, dos que se declararam na raça/cor parda, com 53\%, moradores da zona urbana (83\%), e que não declararam sua escolaridade (28,6\%).

Dados de maior prevalência em indivíduos do sexo masculino também foram encontrados em outros estudos ${ }^{(10-11)}$, que justificaram esses achados pela baixa procura por assistência de saúde, falta de adoção de práticas preventivas, além da presença de fatores de risco, como institucionalização, tabagismo e etilismo. Em outra pesquisa ${ }^{(12)}$ também foi encontrada a associação com a privação de liberdade pelo cárcere.

Quanto à faixa etária, a Tabela 2 expressa que o estudo identificou maior número de notificações com idades entre 25 e 34 anos. Convém enfatizar que estudo semelhante ${ }^{(13)}$ corrobora esses dados, ao identificar 51.088 casos de TB, de um total de 117.638, nessa mesma faixa etária, na região Nordeste, entre os anos de 2015 e 2019. A fim de compreender os valores encontrados, outro achado na literatura ${ }^{(14)}$ menciona que dados assim acompanham o padrão nacional e podem estar associados aos fatores de risco nessa faixa etária, como, por exemplo, a coinfecção com o HIV/Aids. Estudo ${ }^{(15)}$ também menciona como justificativa a alta taxa de abandono do tratamento nessa mesma faixa etária, em decorrência de fatores, como barreiras socioeconômicas, culturais e demográficas, além de aspectos relacionados à própria terapêutica e ao serviço de saúde.

Este estudo também indicou que, na maioria dos casos de TB notificados, a variável escolaridade, bem como a variável relacionada ao tabagismo, não foi declarada, mesmo sendo de registro obrigatório. Para alguns autores ${ }^{(16)}$, este fato pode ser justificado pela possibilidade de serem preenchidas como "ignorado", podendo ainda ser caracterizado como um dado subnotificado, pela falta de clareza nas informações. É importante considerar ainda que, mesmo com a taxa de não declarado, os dados refletem uma predominância da doença em menores níveis de escolaridade, o que corrobora estudo ${ }^{(17)}$ semelhante realizado no Brasil, que ainda relaciona à pobreza desses grupos populacionais, associando variáveis como escolaridade e raça/cor à susceptibilidade ao adoecimento pelo TB.

Ressalta-se ainda que a baixa escolaridade é fator determinante de menor percepção da doença, da falta de conhecimento sobre sua gravidade, e também quanto às possibilidades de acesso ao tratamento, mesmo sendo disponibilizado pelo sistema de saúde brasileiro ${ }^{(13)}$, pois existem outros fatores que demarcam esse acesso.

Houve ainda predominância de casos notificados de residentes na zona urbana, não havendo diferença do reportado na literatura ${ }^{(15,18-19)}$. Este fato também pode ser explicado pela elevada densidade demográfica da zona urbana em relação à rural. Pesquisa ${ }^{(20)}$ fez referência aos indivíduos que vivem em situação de rua na zona urbana e, como mencionado, também apresenta a associação da determinação social com a TB, enfatizando fatores como alimentação inadequada, ingesta de água não potável, exposição em atividades laborais repletas de risco e, mais ainda, o fato de não procurar pelos serviços de saúde, como limitantes à saúde e facilitadores do adoecimento.

Estudo $^{(21)}$ realizado em município da região Norte do Brasil reforça a associação da vulnerabilidade social com a TB e sustenta que o entendimento das formas como a doença é propagada e as ações de saúde são implementadas e impactam na situação de saúde e doença de indivíduos e populações pode repercutir diretamente nas estratégias de planejamento focadas na diversidade.

Como exposto no Gráfico 2, é notório que a forma clínica de TB mais prevalente na região 
Centro-Oeste, no período analisado, foi a pulmonar, o que confirma sua importância epidemiológica, justificada pela predileção do bacilo de Koch pelas áreas com alta concentração de oxigênio, como é o pulmão, e também por sua forma de transmissão facilitar a infecção de indivíduos que são contatos de doentes bacilíferos ${ }^{(22)}$.

Neste estudo, nos dados relativos à soropositividade ao HIV, a proporção de indivíduos testados e com resultado negativo foi de 60,4\% dos casos. Vale mencionar que se identificou ainda uma porcentagem de indivíduos não testados $(26,7 \%)$. Estudo ${ }^{(23)}$ realizado no Nordeste brasileiro encontrou resultados semelhantes, o que é preocupante, devido a persistirem pacientes bacilíferos sem realizar a testagem para HIV, exame que tem importante recomendação para sua realização em todos os novos diagnósticos de TB.

No que tange ao tipo de entrada dos casos de TB no Sistema de Informação de Agravos de Notificação (Sinan), observou-se maior percentual de casos novos $(82,6 \%)$ no período analisado. Estes dados são reforçados por estudo ${ }^{(17)}$ semelhante, que ainda menciona o déficit de diagnóstico ou tratamento como possível interpretação para esses achados. Pode-se destacar também os casos de reingresso, que apareceram de forma menos expressiva. Entretanto, é relevante considerá-los, pois, segundo mencionado na literatura $^{(24)}$, podem enquadrar-se nos casos de pacientes que desenvolveram a TB multirresistente e necessitam de acompanhamento mais rigoroso por parte dos profissionais de saúde, para garantir a qualidade da assistência e do tratamento. Pela elevada taxa de casos novos na região Centro-Oeste nos últimos 10 anos, é preciso considerar a necessidade da realização de maiores esforços, por parte de gestores e profissionais, para acelerar a redução desses números.

O estudo apresenta como limitações o uso de dados secundários, que pode envolver a questão do não preenchimento de algumas variáveis que constam na ficha de notificação compulsória, e o fato de a busca de dados ter sido realizada apenas na plataforma TabNet (DATASUS), o que impossibilitou o acesso a outras informações relevantes, como profissão/ocupação e motivo de desfecho. Insta salientar que, para muitos autores, a questão da subnotificação é fator de distorção da realidade de saúde de populações pesquisadas.

\section{Conclusão}

Este estudo proporcionou a análise da caracterização dos casos notificados de TB na região Centro-Oeste, no período de 2010 a 2019, e concluiu que se assemelham aos encontrados na literatura, reforçando que a TB é uma doença infecciosa atual e sua erradicação ainda exige muito trabalho e políticas públicas efetivas.

Os casos notificados possuíam a caracterização sociodemográfica pertencente, em sua maioria, ao sexo masculino, raça/cor parda, com idade entre 25 e 34 anos, sendo a maioria dos indivíduos moradores na zona urbana. É oportuno salientar que se observou ainda um percentual de indivíduos com escolaridade e tabagismo não declarados no momento da notificação.

Com relação às variáveis clínico-epidemiológicas, foi possível constatar que grande parte dos sujeitos notificados apresentou a forma clínica pulmonar. Do total de casos, mais da metade registrou testagem negativa para o HIV, com um número expressivo de notificações de casos novos.

Os resultados deste trabalho sugerem novos estudos para melhor caracterização desses pacientes bacilíferos e, ainda, pesquisas que explorem a necessidade de realização de programas e ações de prevenção e controle da TB em unidades da Atenção Básica em Saúde, oferecendo subsídios para a tomada de decisão em saúde.

\section{Colaborações:}

1 - concepção, projeto, análise e interpretação dos dados: Yasmin Souza Silva e Paulienne Ramos da Silva Matias;

2 - redação do artigo e revisão crítica relevante do conteúdo intelectual: Yasmin Souza Silva, Paulienne Ramos da Silva Matias, Lucíola Silva Sandim e Maria Goretti Queiroz;

3 - aprovação final da versão a ser publicada: Paulienne Ramos da Silva Matias, Lucíola Silva Sandim, Maria Goretti Queiroz e Jeovana Romero de Serqueira. 
Yasmin Souza Silva, Paulienne Ramos da Silva Matias,

\section{Referências}

1. Brasil. Ministério da Saúde. Guia de vigilância epidemiológica [Internet]. Brasília, DF; 2009 [cited 2020 Jun 17]. Available from: https://bvsms. saude.gov.br/bvs/publicacoes/guia_vigilancia_ epidemiologica_7ed.pdf

2. World Health Organization. The End TB Strategy [Internet]. Genebra (CHE); 2015 [cited 2020 Oct 13]. Available from: https://www.who.int/tb/strategy/ en/

3. World Health Organization. Global Tuberculosis Report 2018 [Internet]. Genebra (CHE); 2018 [cited 2020 Oct 13]. Available from: https://apps.who.int/ iris/handle/10665/274453

4. Brasil. Ministério da Saúde. Brasil Livre da Tuberculose: Plano Nacional pelo Fim da Tuberculose como Problema de Saúde Pública [Internet]. Brasília (DF); 2017 [cited 2020 Jun 17]. Available from: http://bvsms.saude.gov.br/bvs/ publicacoes/brasil_livre_tuberculose_plano_ nacional.pdf

5. World Health Organization. Global Tuberculosis Report 2020 [Internet]. Genebra (CHE); 2020 [cited 2021 Jul 31]. Available from: https://www.who.int/ publications/i/item/9789240013131/

6. Brasil. Ministério da Saúde. SINAN - Sistema de Informação de Agravos de Notificação [Internet]. Brasília (DF); 2020 [cited 2020 Sep 4]. Available from: http://www.datasus.gov.br

7. Brasil. Ministério da Saúde. Manual de Recomendações para o Controle da Tuberculose no Brasil [Internet]. 2a ed. Brasília (DF); 2019 [cited 2020 Jun 17]. Available from: https://bvsms.saude. gov.br/bvs/publicacoes/manual_recomendacoes_ controle_tuberculose_brasil_2_ed.pdf

8. Paula WKAS, Samico IC, Caminha MFC, Batista Filho M, Silva SL. Primary health care assessment from the users' perspectives: a systematic review. Rev Esc Enferm USP. 2016;50(2):331-40. DOI: http://dx.doi.org/10.1590/ S0080-623420160000200021

9. Instituto Brasileiro de Geografia e Estatística. Estimativas Populacionais 2020 [Internet]. Rio de Janeiro; 2020 [cited 2020 Oct 13]. Available from: https://www.ibge.gov.br/estatisticas/sociais/ populacao/9103-estimativas-de-populacao.html?= $\& \mathrm{t}=\mathrm{O}-$ que-e

10. Santos TA, Martins MMF. Perfil dos casos de reingresso após abandono do tratamento da tuberculose em Salvador, Bahia, Brasil. Cad Saúde Coletiva. 2018;26(3):233-40. DOI: 10.1590/1414-462X201800030235

11. Santos BO, Brito TVR, Mesquita CR, Guimarães RJPS, Leão LA, Rocha MP. Space-temporal analysis of the incidence of tuberculosis in primary care. Pará Res Med J. 2018;1(2):e21. DOI: 10.4322/prmj.2017.021

12. Carbone ASS, Sgarbi RVE, Lemos EF, Paião DSG, Simionatto S, Castro ARCM, et al. Estudo multicêntrico da prevalência de tuberculose e HIV na população carcerária do Estado do Mato Grosso do Sul. Com Ciências Saúde [Internet]. 2017 [cited 2020 Oct 20]; 28(1):53-7. Available from: http://bvsms.saude.gov. br/bvs/periodicos/ccs_artigos/estudo_multicentrico.pdf

13. Sousa GO, Sales BN, Gomes JGF, Silva MA, Oliveira GAL. Tuberculosis epidemiology in northeastern Brazil, 2015-2019. Res Soc Dev. 2020;9(8):e:82985403 DOI: https://doi.org/10.33448/ rsd-v9i8.5403

14. Barioto JG, Anversa L. Perfil epidemiológico dos casos de tuberculose notificados no município de Bauru, Estado de São Paulo, Brasil. Bol Epidemiol Paulista [Internet]. 2015 [cited 2020 Oct 28];12(134):1-11. Available from: https:// www.researchgate.net/publication/291126460_ Perfil_epidemiologico_dos_casos_de_tuberculose_ notificados_no_municipio_de_Bauru_estado_de_ Sao_Paulo_Brasil

15. Jesus GAS, Reis IM, Miranda ML, Silva MR. Acompanhamento e situação de encerramento de casos de tuberculose notificados. Rev enferm UFPE. 2021;15(1):e246020. DOI: https://doi. org/10.5205/1981-8963.2021.246020

16. Bartholomay P, Pinheiro RS, Pelissari DM, Arakaki-Sanchez D, Dockhorn F, Rocha JL, et al. Sistema de Informação de Tratamentos Especiais de Tuberculose (SITE-TB): histórico, descrição e perspectivas. Epidemiol Serv Saúde. 2019;28(2): e2018158. DOI: http://dx.doi.org/10.5123/s167949742019000200002

17. Fontes GJF, Silva TG, Sousa JCM, Feitosa ANA, Silva ML, Bezerra ALD, et al. Perfil epidemiológico da tuberculose no Brasil no período de 2012 a 2016. Rev bras educ e saúde. 2019;9(1):19-26. DOI: https://doi.org/10.18378/rebes.v9i1.6376

18. Queiroga RPF, Sá LD, Nogueira JA, Lima ERV, Silva ACO, Pinheiro PGOD, et al. Distribuição espacial da tuberculose e a relação com condições 
de vida na área urbana do município de Campina Grande - 2004 a 2007. Rev Bras Epidemiol. 2012;15(1):222-32. DOI: http://dx.doi.org/10.1590/ S1415-790X2012000100020

19. Arroyo LH, Yamamura M, Protti-Zanatta ST, Fusco APB, Palha PF, Ramos ACV, et al. Identificação de áreas de risco para a transmissão da tuberculose no município de São Carlos, São Paulo, 2008 a 2013. Epidemiol Serv Saúde. 2017;26(3):525-34. DOI: http://dx.doi.org/10.5123/ s1679-49742017000300010

20. Zuim RCB, Trajman A. Itinerário terapêutico de doentes com tuberculose vivendo em situação de rua no Rio de Janeiro. Physis: Rev Saúde Coletiva. 2018;28(2):e280205 DOI: http://dx.doi. org/10.1590/s0103-73312018280205

21. André SR, Nogueira LMV, Rodrigues ILA, Cunha TN, Palha PF, Santos CB. Tuberculosis associated with the living conditions in an endemic municipality in the North of Brazil. Rev Latino-Am Enfermagem. 2020;28:e3343. DOI: http://dx.doi. org/10.1590/1518-8345.3223.3343
22. Silva LT, Felipini MCC, Oliveira TB, Brunello MEF, Órfão NH. Perfil epidemiológico da tuberculose no serviço de referência do estado de Rondônia. Rev Epidemiol Controle Infec. 2019;9(1):48-54. DOI: https://doi.org/10.17058/reci.v9i1.12249

23. Barreto MTS, Santos GM, Monteiro MJDSD, Jesus RLR, Barbosa GS, Oliveira VA. Epidemiology of tuberculosis in a northeast brazilian state. Res Soc Develop. 2020;9(7):e52973643. DOI: https://doi.org/10.33448/rsd-v9i7.3643

24. Farias EJSF, Albuquerque IMN, Araújo RA, Soares JSA, Linhares MSC. Análise epidemiológica dos casos de tuberculose notificados no município de Sobral - CE no período de 2007 a 2011. Sanare (Sobral, Online). [Internet]. 2013 [cited 2020 Oct 20];12(1):33-9. Available from: https://sanare. emnuvens.com.br/sanare/article/view/333

Recebido: 14 de janeiro de 2021

Aprovado: 22 de outubro de 2021

Publicado: 4 de fevereiro de 2022

A Revista Baiana de Enfermagem utiliza a Licença Creative Commons - Atribuição-NãoComercial 4.0 Internacional.

https://creativecommons.org/licenses/by-nc/4.0/

Este artigo é de acesso aberto distribuído sob os termos da Licença Creative Commons (CC BY-NC).

Esta licença permite que outros remixem, adaptem e criem a partir do seu trabalho para fins não comerciais. Embora os novos trabalhos tenham de lhe atribuir o devido crédito e não possam ser usados para fins comerciais, os usuários não têm de licenciar esses trabalhos derivados sob os mesmos termos. 